\title{
Quantum Andreev Map: A Paradigm of Quantum Chaos in Superconductivity
}

\author{
Ph. Jacquod, ${ }^{1}$ H. Schomerus, ${ }^{2}$ and C.W. J. Beenakker ${ }^{1}$ \\ ${ }^{1}$ Instituut-Lorentz, Universiteit Leiden, P.O. Box 9506, 2300 RA Leiden, The Netherlands \\ ${ }^{2}$ Max Planck Institute for the Physics of Complex Systems, Nöthnitzer Strasse 38, 01187 Dresden, Germany \\ (Received 20 December 2002; revised manuscript received 24 March 2003; published 22 May 2003)
}

\begin{abstract}
We introduce quantum maps with particle-hole conversion (Andreev reflection) and particle-hole symmetry, which exhibit the same excitation gap as quantum dots in the proximity to a superconductor. Computationally, the Andreev maps are much more efficient than billiard models of quantum dots. This makes it possible to test analytical predictions of random-matrix theory and semiclassical chaos that were previously out of reach of computer simulations. We have observed the universal distribution of the excitation gap for a large Lyapunov exponent and the logarithmic reduction of the gap when the Ehrenfest time becomes comparable to the quasiparticle dwell time.
\end{abstract}

DOI: 10.1103/PhysRevLett.90.207004

One-dimensional (1D) quantum mechanical models with a chaotic classical limit were first studied by Casati, Chirikov, Ford, and Izrailev in 1979 [1]. These models have since developed into one of two paradigms of quantum chaos [2,3]. The other paradigm is the $2 \mathrm{D}$ billiard of irregular shape [4]. Two is the lowest number of dimensions for nonintegrable (chaotic) dynamics in autonomous systems, since a single constant of motion is sufficient for integrability in 1D. The 1D models get around this constraint through a periodically timedependent external force ("kick"), which eliminates the energy as a constant of motion-but still conserves the quasienergy (analogously to quasimomentum conservation in a periodic lattice). The two paradigms share a common set of phenomena in the fields of quantum chaos and localization [5-8].

The combination of chaos and superconductivity produces an entirely new phenomenology, notably the appearance of an excitation gap as a signature of quantum chaos [9]. The paradigm common to most of the literature is the $2 \mathrm{D}$ billiard connected to a superconductor [10], introduced under the name "Andreev billiard" in Ref. [11]. The name refers to the Andreev reflection which occurs at the interface with the superconductor, where an electron at energy $\varepsilon$ above the Fermi level is converted into a hole at energy $\varepsilon$ below it.

From the point of view of computational efficiency, compact quantum maps such as the kicked rotator [1] (a particle confined to a circle and driven periodically in time with a strength that depends on its position) are much more powerful than 2D models such as billiards. Indeed, there exists a highly efficient diagonalization technique that works only for maps [12]. The lack of a 1D map for quantum chaos with superconductivity has hindered the numerical test of a variety of analytical predictions [13-20]. Most notably, numerical efforts have not been able to distinguish the conflicting predictions [9] of random-matrix theory (RMT) and the semiclassical Bohr-Sommerfeld (BS) quantization: RMT predicts an excitation gap at the Thouless energy while
PACS numbers: 74.45.+c, 05.45.Mt, 73.23.-b, 74.78.Na

BS gives an exponentially vanishing density of states without a true gap. Recent analytical work [16-20] has predicted that diffraction creates a gap in the BS density of states at the inverse Ehrenfest time. This has never been seen in computer simulations, because the Ehrenfest time scales logarithmically with the system size and is usually far too small to play a role. For these reasons there is a real need for something like a "quantum Andreev map." Does it exist? If it does, can it be simulated more efficiently than the Andreev billiard? These are the issues addressed in this paper.

We show how to construct quantum Andreev maps out of any conventional quantum map (not necessarily chaotic), in much the same way as any normal billiard can be turned into an Andreev billiard by coupling it to a superconductor. The construction is guided by the classical electron and hole dynamics on the Poincaré surface of section of an Andreev billiard. The Andreev kicked rotator is a particular example of such an Andreev map. We certify that it possesses the phenomenology of the Andreev billiards and search for predictions of RMT and semiclassics. We leave for future investigations the application of the Andreev map to other kicked models (possibly with a different phenomenology), such as the kicked top [3] and the Fermi-Ulam model [21].

A quantum map is represented by the Floquet operator $F$, which gives the stroboscopic time evolution $u\left(p \tau_{0}\right)=$ $F^{p} u(0)$ of an initial wave function $u(0)$. (We set the stroboscopic period $\tau_{0}=1$ in most equations.) The unitary $M \times M$ matrix $F$ has eigenvalues $\exp \left(-i \varepsilon_{m}\right)$, with the quasienergies $\varepsilon_{m} \in(-\pi, \pi)$ (measured in units of $\hbar / \tau_{0}$ ). This describes particle excitations in a normal metal. We also need hole excitations. A particle excitation with energy $\varepsilon_{m}$ (measured relatively to the Fermi level) is identical to a hole excitation with energy $-\varepsilon_{m}$. This means that hole excitations in a normal metal have Floquet operator $F^{*}$ and wave function $v(p)=\left(F^{*}\right)^{p} v(0)$.

Particles and holes are coupled by connecting the normal metal via a lead to a superconducting reservoir. On a Poincare surface of section, the lead is represented 
by a spatially localized region in which Andreev reflection converts electrons into holes and vice versa, with phase shift $-i$. (A weak energy dependence of this phase shift is ignored for simplicity, but can be accounted for straightforwardly.) Analogously, for the quantum Andreev map, we assume that Andreev reflections occur whenever an excitation ends up in a certain subspace of Hilbert space. This subspace $n_{1}, n_{2}, \ldots, n_{N}$ consists of $N$ out of $M$ states in a chosen basis and corresponds to a lead with $N$ propagating channels. The $N \times M$ matrix $P$ projects onto the lead. Its elements are $P_{n m}=1$ if $m=$ $n \in\left\{n_{1}, n_{2}, \ldots, n_{N}\right\}$ and $P_{n m}=0$ otherwise. The dwell time of a quasiparticle excitation in the normal metal is $\tau_{\text {dwell }}=M / N$, equal to the mean time between Andreev reflections. The fact that Andreev reflections occur only at multiples of the stroboscopic time $\tau_{0}$ is technically convenient, and should be physically irrelevant for $\tau_{\text {dwell }} \gg \tau_{0}$.

Putting all this together, we construct the quantum Andreev map from the matrix product

$$
\mathcal{F}=\mathcal{P}\left(\begin{array}{cc}
F & 0 \\
0 & F^{*}
\end{array}\right), \quad \mathcal{P}=\left(\begin{array}{cc}
1-P^{\mathrm{T}} P & -i P^{\mathrm{T}} P \\
-i P^{\mathrm{T}} P & 1-P^{\mathrm{T}} P
\end{array}\right) .
$$

(The superscript " $\mathrm{T}$ " indicates the transpose of a matrix.) The particle-hole wave function $\Psi=(u, v)$ evolves in time as $\Psi(p)=\mathcal{F}^{p} \Psi(0)$. The Floquet operator can be symmetrized (without changing its eigenvalues) by the unitary transformation $\mathcal{F} \rightarrow \mathcal{P}^{-1 / 2} \mathcal{F} \mathcal{P}^{1 / 2}$, with

$$
\mathcal{P}^{1 / 2}=\left(\begin{array}{cc}
1-\left(1-\frac{1}{2} \sqrt{2}\right) P^{\mathrm{T}} P & -i \frac{1}{2} \sqrt{2} P^{\mathrm{T}} P \\
-i \frac{1}{2} \sqrt{2} P^{\mathrm{T}} P & 1-\left(1-\frac{1}{2} \sqrt{2}\right) P^{\mathrm{T}} P
\end{array}\right) .
$$

In order to establish the correspondence of the 1D quantum Andreev maps to 2D Andreev billiards, we examine the spectral properties of the map. The Floquet operator $\mathcal{F}$ possesses a particle-hole symmetry which entails that its $2 M$ eigenvalues $\exp \left(-i \varepsilon_{m}\right)$ come in inverse pairs. This symmetry is the analogue of the particle-hole symmetry in Andreev billiards, in which excitation energies $\pm \varepsilon$ occur symmetrically around the Fermi level. To avoid double counting of levels, we restrict the quasienergy to the interval $(0, \pi)$. The excitation spectrum of particles and holes consists of the $M$ quasienergies in this interval, and the mean level spacing $\pi / M$ is twice as small as the level spacing $\delta=2 \pi / M$ for particles and holes separately. The energy scale for the proximityinduced excitation gap is the Thouless energy $E_{\mathrm{T}}=$ $N \delta / 4 \pi=N / 2 M=1 /\left(2 \tau_{\text {dwell }}\right)$.

The quantization condition $\operatorname{det}\left(\mathcal{F}-e^{-i \varepsilon}\right)=0$ can be written equivalently as

$$
\operatorname{det}\left[1+S(\varepsilon) S^{*}(-\varepsilon)\right]=0,
$$

in terms of the $N \times N$ scattering matrix [22]

$$
S(\varepsilon)=P\left[e^{-i \varepsilon}-F\left(1-P^{\mathrm{T}} P\right)\right]^{-1} F P^{\mathrm{T}} .
$$

Equation (3) for the Andreev map has the same form as for the Andreev billiard [23], but there $S$ is given in terms of a Hamiltonian $H_{0}$ instead of a Floquet operator $F$. The two approaches become entirely equivalent in the context of RMT, when $H_{0}$ is chosen from one of the Gaussian ensembles and $F$ is chosen from one of the circular ensembles [24]. They are also equivalent in the semiclassical limit, when the billiard can be represented by a Poincaré map which can be quantized approximately [25].

In the mean-field limit $M \gg N \gg 1$, RMT predicts a hard gap in the excitation spectrum of size $E_{\mathrm{RMT}}=\gamma E_{\mathrm{T}}$ [with $\gamma=2^{-3 / 2}(\sqrt{5}-1)^{5 / 2}=0.60$ ], and above the gap a square-root singularity in the density of states $\rho(\varepsilon)=$ $\pi^{-1} \Delta^{-3 / 2}\left(\varepsilon-E_{\mathrm{RMT}}\right)^{1 / 2} \quad$ (with $\Delta=0.068 N^{1 / 3} \delta$ ) [9]. Sample-to-sample fluctuations of the lowest excitation energy $\varepsilon_{0}$ around the mean-field gap have been calculated in Refs. [13,14]. A universal probability distribution was predicted for the rescaled energy $x=\left(\varepsilon_{0}-E_{\mathrm{RMT}}\right) / \Delta$. While the mean-field prediction of RMT has been tested numerically in an Andreev billiard [9], the numerical error bars are too large to extract the predicted universal gap fluctuations.

To demonstrate the efficiency of the quantum Andreev maps, we specialize to the quantum kicked rotator. The Floquet operator is [2]

$$
F=\exp \left(i \frac{\hbar \tau_{0}}{4 I_{0}} \frac{\partial^{2}}{\partial \theta^{2}}\right) \exp \left(-i \frac{K I_{0}}{\hbar \tau_{0}} \cos \theta\right) \times \exp \left(i \frac{\hbar \tau_{0}}{4 I_{0}} \frac{\partial^{2}}{\partial \theta^{2}}\right),
$$

with $I_{0}$ the moment of inertia of the particle and $K$ the (dimensionless) kicking strength. The particle moves freely along the circle for half a period, is then kicked with a strength $K \cos \theta$, and proceeds freely for another half period. The transition from classical to quantum behavior is governed by the effective Planck constant $\hbar_{\text {eff }} \equiv \hbar \tau_{0} / I_{0}$. Since we would like to compare the kicked rotator to a chaotic billiard, without localization, we follow the usual procedure of quantizing phase space on the torus $\theta, p \in(0,2 \pi)$, rather than on a cylinder, with $p=-i \hbar_{\text {eff }} \partial / \partial \theta$ the dimensionless angular momentum [2]. For $\hbar_{\mathrm{eff}}=2 \pi / M$, with integer $M$, the Floquet operator is an $M \times M$ unitary symmetric matrix. In angular momentum representation it has elements

$$
\begin{aligned}
F_{k k^{\prime}} & =e^{-(i \pi / 2 M)\left(k^{2}+k^{\prime 2}\right)}\left(U Q U^{\dagger}\right)_{k k^{\prime}}, \\
U_{k k^{\prime}} & =M^{-1 / 2} e^{(2 \pi i / M) k k^{\prime}}, \\
Q_{k k^{\prime}} & =\delta_{k k^{\prime}} e^{-(i M K / 2 \pi) \cos (2 \pi k / M)} .
\end{aligned}
$$

Upon increasing $K$, the classical dynamics varies from fully integrable $(K=0)$ to fully chaotic $[K \gtrsim 7$, with Lyapunov exponent $\lambda \approx \ln (K / 2)]$. For $K<7$, stable and unstable motion coexist (a so-called mixed phase space).

To introduce the Andreev reflection, we use a projection operator which is diagonal in $p$ representation,

$$
\left(P^{\mathrm{T}} P\right)_{k k^{\prime}}=\delta_{k k^{\prime}} \times \begin{cases}1 & \text { if } L \leq k \leq L+N-1 \\ 0 & \text { otherwise. }\end{cases}
$$


(We checked that similar results are obtained when $P$ is diagonal in $\theta$ representation.) The position $L$ of the lead to the superconductor is arbitrary. The Floquet operator $\mathcal{F}$ of the "Andreev kicked rotator" is then obtained by inserting Eqs. (6) and (7) into Eq. (1). We apply the symmetrization (2), so $\mathcal{F}$ is a unitary symmetric matrix. The real symmetric matrix $\frac{1}{2}\left(\mathcal{F}+\mathcal{F}^{\dagger}\right)$ can be diagonalized efficiently with $\mathcal{O}\left(M^{2} \ln M\right)$ operations [and not $\mathcal{O}\left(M^{3}\right)$ as with standard methods] by means of the Lanczos technique, if the multiplication with the matrix $U$ is carried out with the help of the fast Fourier transform algorithm [12]. The eigenvalues $\cos \varepsilon_{m}$ uniquely determine the quasienergy $\varepsilon_{m} \in(0, \pi)$.

In the inset of Fig. 1, we show the density of states $\rho(\varepsilon)$ for system size $M=8192$, kicking strength $K=45$ (strongly chaotic dynamics), and several widths $N$ of the lead to the superconductor. The density of states has been averaged over 250 different positions of the lead. The data points fall on top of the RMT prediction [9] without any adjustable parameter. Reducing the kicking strength down to $K=1.2$, one enters the regime of mixed classical dynamics. We see that the gap disappears, as predicted in Ref. [26].

To test RMT beyond the mean-field limit, we study the statistical fluctuations of the gap. The main panel of Fig. 1 shows the probability distribution of the smallest eigenvalue $\varepsilon_{0}$ in the chaotic regime. To improve statistics we sampled 6000 different positions of the lead. We rescaled the energy $x=\left(\varepsilon_{0}-E_{\mathrm{RMT}}\right) / \Delta$, as prescribed by Ref. [13]. Good agreement is observed with the universal scaling distribution [27], again without any adjustable parameters.

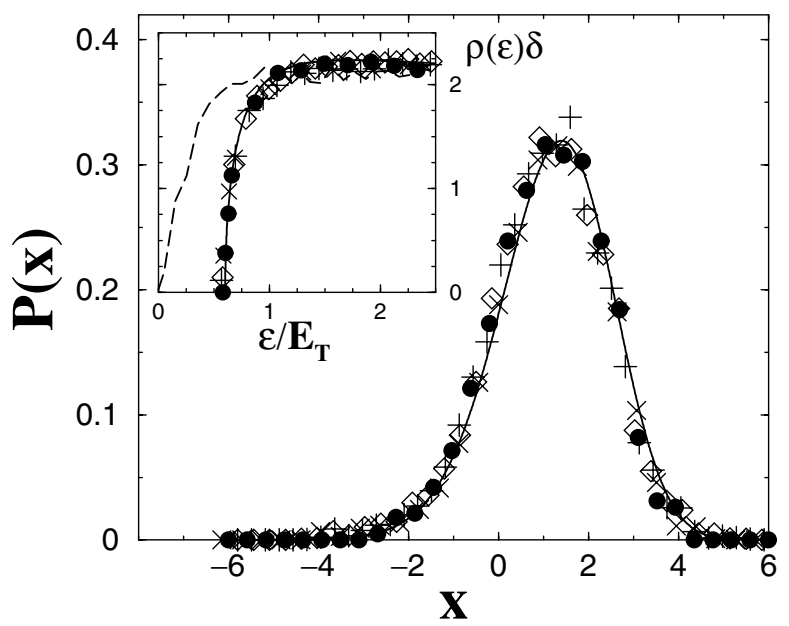

FIG. 1. Main plot: Gap distribution for the Andreev kicked rotator with $M=8192, K=45$, and $M / N=\tau_{\text {dwell }}=10$ (diamonds), 20 (circles), $40(+)$, and $50(\times)$. The solid line gives the RMT prediction [13]. Inset: Density of states for the same system. The solid line is the RMT prediction [9]. The dashed line is a numerical result in the mixed regime $(M=8192, K=$ $1.2, M / N=10)$.
It is predicted theoretically that deviations from RMT should appear if the Ehrenfest time $\tau_{\mathrm{E}}=\lambda^{-1} \ln M$ is no longer small compared to $\tau_{\mathrm{dwell}}$. For $\tau_{\mathrm{E}} \gtrsim \tau_{\mathrm{dwell}}$, the semiclassical Bohr-Sommerfeld approximation $[9,26]$ should be valid, with a diffraction induced gap of the order of $\hbar / \tau_{\mathrm{E}}$ [16]. To search for these deviations from RMT, we consider rotators with smaller kicking strengths (but still in the fully chaotic regime), thus smaller Lyapunov exponent, and much larger $M$.

In Fig. 2, we show the density of states for $M=$ 131072 and $K=14$. Strong deviations from the RMT prediction are clearly visible. Also plotted is the result of a BS calculation [9], in which we slightly smoothed the singular delta functions. This approximation agrees better with the exact result. Most remarkably, it reproduces the three distinct peaks in the density of states, which now can be identified with trajectories of certain lengths. All trajectories with lengths that are odd multiples of $\tau_{\text {dwell }}=$ 5 contribute to the peak at $\varepsilon / E_{\mathrm{T}}=\pi$, odd multiples of 4 contribute at $\varepsilon / E_{\mathrm{T}}=5 \pi / 4$, and odd multiples of 3 contribute at $\varepsilon / E_{\mathrm{T}}=5 \pi / 3$.

A systematic reduction of the excitation gap is observed upon increasing the ratio $\tau_{\mathrm{E}} / \tau_{\mathrm{dwell}}$, as shown in Fig. 3 . The main panel is a semilogarithmic plot of $\varepsilon_{0} / E_{T}$ as a function of $M \in\left[2^{9}, 2^{19}\right]$, for $M / N=\tau_{\text {dwell }}=5$ and $K=14$. Existing theories $[19,20]$ predict a linear initial decrease of $\varepsilon_{0}$ with $\ln M$ at fixed $\tau_{\text {dwell }}=M / N$. We fit the data to the prediction of Vavilov and Larkin [20],

$$
\frac{\varepsilon_{0}}{E_{\mathrm{RMT}}}=1-\frac{\alpha}{2 \lambda \tau_{\mathrm{dwell}}}\left(\ln M-2 \ln \frac{M}{N}-\alpha^{\prime}\right) .
$$

We find $\alpha=0.59$ and $\alpha^{\prime}=3.95$. Once $\alpha$ and $\alpha^{\prime}$ are extracted, no free parameter is left, and the resulting curve, shown with a solid line in the inset of Fig. 3, correctly reproduces the parametric dependence on

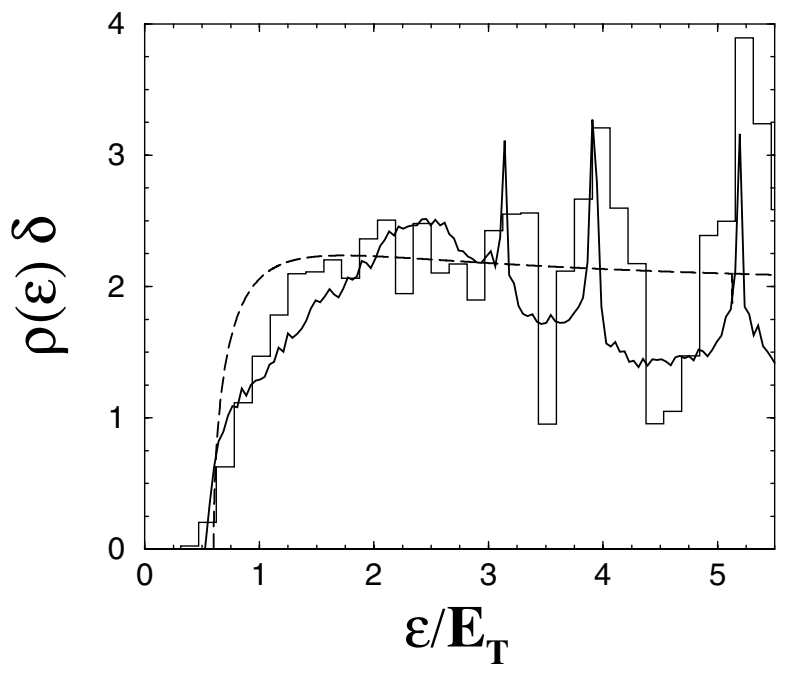

FIG. 2. Density of states for the Andreev kicked rotator with $M=131072, \tau_{\text {dwell }}=5$, and $K=14$ (solid line), compared with the Bohr-Sommerfeld calculation (histogram), and the RMT prediction (dashed line). 


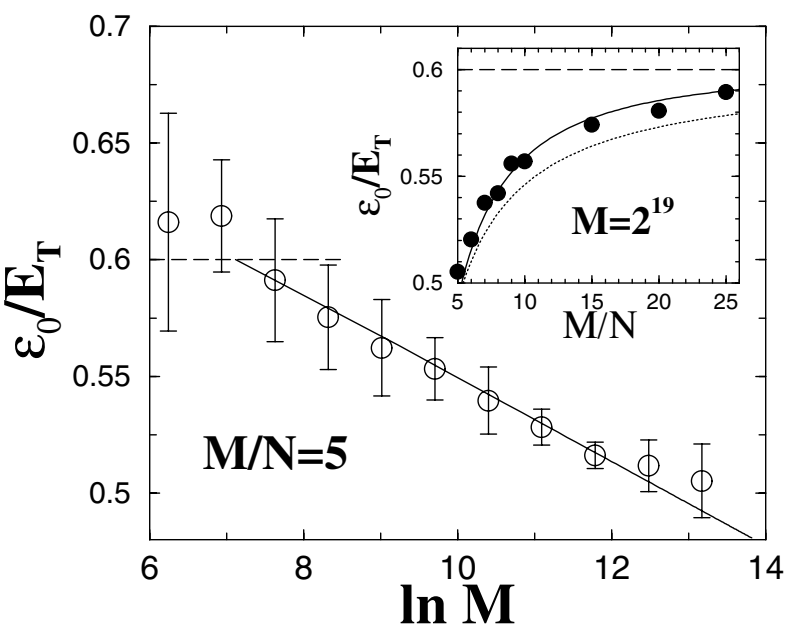

FIG. 3. Main plot: Dependence of the mean gap on the system size $M$, for $\tau_{\text {dwell }}=M / N=5$ and $K=14$. Averages have been calculated with 400 (for $M=512$ ) to 40 (for $M>5 \times 10^{5}$ ) different positions of the contacts to the superconductor. The error bars represent the root mean square of $\varepsilon_{0}$. The dashed line is the RMT prediction and the solid line is a linear fit to the data points. Inset: Dependence of the mean gap on $\tau_{\text {dwell }}=$ $M / N$ for $K=14$ and $M=524288$. The dashed line is the RMT prediction and the solid and dotted curves are given by Eqs. (8) and (9), respectively, with coefficients extracted from the linear fit in the main plot.

$\tau_{\text {dwell }}=M / N$ at fixed $M$. As a further check, we tried the slightly different expression

$$
\frac{\varepsilon_{0}}{E_{\mathrm{RMT}}}=1-\frac{\alpha}{2 \lambda \tau_{\mathrm{dwell}}}\left(\ln M-\alpha^{\prime \prime}\right),
$$

with $\alpha^{\prime \prime}=\alpha^{\prime}+2 \ln 5$. The resulting curve (dotted line in the inset of Fig. 3) shows significant deviations from the numerical data. We conclude that Eq. (8) gives the correct parametric dependence of the Andreev gap.

A discrepancy remains in the value of the numerical coefficients. While the coefficient $\alpha^{\prime}$ is model dependent, the prefactor $\alpha$ is expected to be universal. Our numerics gives $\alpha=0.59 \pm 0.08$, in between the two competing predictions $\alpha=0.23$ of Ref. [20] and $\alpha=2$ of Ref. [19].

In conclusion, we have constructed a quantum map that accounts for the presence of superconductivity. The Andreev kicked rotator introduced above has been shown to be equivalent to the Andreev billiards studied thus far. Owing to the fact that it is one dimensional rather than two dimensional, it is much more efficient computationally, which permits one to observe two theoretical predictions that are currently out of reach of billiard simulations: the universal gap fluctuations for a large Lyapunov exponent and the logarithmic reduction of the gap for a small Lyapunov exponent. We foresee that the Andreev kicked rotator on a cylinder (instead of on a torus) can be an equally effective tool to study the interplay of superconductivity and localization.
We have benefitted from discussions with İ. Adagideli and J. Tworzydło. This work was supported by the Dutch Science Foundation NWO/FOM.

[1] G. Casati, B. V. Chirikov, J. Ford, and F. M. Izrailev, Lect. Notes Phys. 93, 334 (1979).

[2] F. M. Izrailev, Phys. Rep. 196, 299 (1990).

[3] F. Haake, Quantum Signatures of Chaos (SpringerVerlag, Berlin, 1992).

[4] O. Bohigas, M.-J. Giannoni, and C. Schmit, Phys. Rev. Lett. 52, 1 (1984).

[5] S. Fishman, D. R. Grempel, and R. E. Prange, Phys. Rev. Lett. 49, 509 (1982).

[6] A. Altland and M. R. Zirnbauer, Phys. Rev. Lett. 77, 4536 (1996).

[7] F. Borgonovi, G. Casati, and B.W. Li, Phys. Rev. Lett. 77, 4744 (1996).

[8] K. M. Frahm and D. L. Shepelyansky, Phys. Rev. Lett. 79, 1833 (1997).

[9] J. A. Melsen, P.W. Brouwer, K. M. Frahm, and C.W. J. Beenakker, Europhys. Lett. 35, 7 (1996); Phys. Scr. T69, 223 (1997).

[10] An exception is the study of Andreev reflection in the framework of quantum graphs by S. Gnutzmann, B. Seif, F. von Oppen, and M. R. Zirnbauer, Phys. Rev. E 67, 046225 (2003).

[11] I. Kosztin, D. L. Maslov, and P. M. Goldbart, Phys. Rev. Lett. 75, 1735 (1995).

[12] R. Ketzmerick, K. Kruse, and T. Geisel, Physica (Amsterdam) 131D, 247 (1999).

[13] M. G. Vavilov, P.W. Brouwer, V. Ambegaokar, and C. W. J. Beenakker, Phys. Rev. Lett. 86, 874 (2001).

[14] P. M. Ostrovsky, M. A. Skvortsov, and M.V. Feigelman, Phys. Rev. Lett. 87, 027002 (2001); JETP Lett. 75, 336 (2002).

[15] A. Lamacraft and B. D. Simons, Phys. Rev. B 64, 014514 (2001).

[16] A. Lodder and Yu.V. Nazarov, Phys. Rev. B 58, 5783 (1998).

[17] D. Taras-Semchuk and A. Altland, Phys. Rev. B 64, 014512 (2001)

[18] İ. Adagideli and C.W. J. Beenakker, Phys. Rev. Lett. 89, 237002 (2002).

[19] P. G. Silvestrov, M. C. Goorden, and C.W. J. Beenakker, Phys. Rev. Lett. 90, 116801 (2003).

[20] M. G. Vavilov and A. I. Larkin, Phys. Rev. B 67, 115335 (2003).

[21] S. R. Jain, Phys. Rev. Lett. 70, 3553 (1993).

[22] A. Ossipov, T. Kottos, and T. Geisel, cond-mat/0208378.

[23] K. M. Frahm, P.W. Brouwer, J. A. Melsen, and C.W. J. Beenakker, Phys. Rev. Lett. 76, 2981 (1996).

[24] Y.V. Fyodorov and H.-J. Sommers, JETP Lett. 72, 422 (2000).

[25] E. B. Bogomolny, Nonlinearity 5, 805 (1992).

[26] H. Schomerus and C. W. J. Beenakker, Phys. Rev. Lett. 82, 2951 (1999).

[27] C. A. Tracy and H. Widom, Commun. Math. Phys. 159, 151 (1994). 